\title{
Game-playing as an effective learning resource for elderly people: encouraging experiential adoption of touchscreen technologies
}

\author{
Sabrina Oppl ${ }^{1} \cdot$ Christian Stary $^{1}$
}

Published online: 3 November 2018

(c) The Author(s) 2018

\begin{abstract}
Elderly people often struggle to keep up with digital advancements, particularly given the fast pace of such developments and the increasing need to interact with novel devices in everyday life. To include elderly people in ongoing socio-technological developments, we aim to overcome the barriers to learning and adoption such older adults may face. In this contribution, we set the methodological stage for an interactive game-playing approach using touchscreen tablets. By using games in learning settings, we encourage elderly people in digital adoption and skill development. In a first study, a video analysis of game-playing sessions and active learning support were performed. Although approaches that combine game-playing with learning could encourage elderly people to become an integral part of a technologically advancing society, the facilitation of interaction processes in individual and collective gaming seems to be crucial. We report on the performed field study and provide methodological suggestions for investigating digital media adoption.
\end{abstract}

Keywords Ageing $\cdot$ Elderly people $\cdot$ Digital adoption $\cdot$ Facilitation $\cdot$ Learning $\cdot$ Game-playing $\cdot$ UTAUT2

\section{Introduction}

Today, age is closely linked with the ability or inability to cope with technological changes, in particular when an individual aims to be an active member of the information and communication society [1]. The older population is a heterogeneous group [2] with differences in access to digital information [3]. While digital adoption is rising also in this group [4], the digital spectrum [5] still includes a group of older citizens that tend to be unfamiliar with the use of new digital devices [6]. Keeping up with rapidly developing technologies and the adjacent pace of life can be quite challenging for them [7, 8]. Prominent individual obstacles are causing a decline in motivation to learn and less capability to learn. These are compounded by certain barriers to learning, which include negative stereotypes about elderly people

Sabrina Oppl

sabrina.oppl@jku.at

https://www.jku.at/ce/content

Christian Stary

christian.stary@jku.at

1 Department of Business Informatics-Communications Engineering, Johannes Kepler University Linz, Science Park 3, Altenberger Straße 69, 4040 Linz, Austria and a lack of a supportive learning climate for people over a certain age [9].

Research suggests that the more active and educated older adults are, "the less drain they are on family and community resources and services" [10]. Further, it has been acknowledged that "active and healthy [elderly people] contribute to community wellbeing through their accumulated life experience, expertise, and service" [11], and so the relationship between lifelong learning and community wellbeing can be argued from a social capital perspective [12]. Therefore, research on the factors that contribute to the acquisition of digital knowledge can provide novel approaches and could contribute to new insights for supporting learning and engagement process management of an increasingly older population. ${ }^{1}$

Game-playing is one possible approach to supporting older people to deal and engage with new digital technologies. Studies show that elderly individuals possess "a great potential to accept" [13] and play digital games, which can help them to acquire digital skills that are transferable to and useful in everyday life [13, 14]. As pedagogical tools,

\footnotetext{
1 Society is getting older, as the average age of populations continues to rise substantially in the next several decades, as reported in the United Nations World Aging Population 2017 report (http://www. un.org/en/development/desa/population/publications/pdf/ageing/ WPA2017_Highlights.pdf).
} 
games have gained significance, as there is increasing realisation that they "invoke images and feelings of a challenge, a reward or excitement of the unknown" [15]. Brooks [12] has framed game-playing through a tangible context for elderly people, and also found positive effects of gaming when acquiring digital skills. However, existing research suggests that support measures are essential if older individuals are to eventually learn to deal with new technologies $[6,10,16,17]$.

In the following, we report on a European initiative to combine game-playing with learning and supportive activities in order to promote the adoption of interactive media by elderly people. This initiative is manifest in the Erasmus+ project GIRDA (Gameplay for Inspiring Digital Adoption). The overall objective of the project is to overcome the barriers to learning and adoption faced by older adults, and thereby help to close the digital divide that exists between generations. Digital adoption refers to technical adoption, which is defined as "a process", one that starts "with the user becoming aware of the technology, and [ends] with the user embracing the technology and making full use of it" [18]. In this way, the term highlights the focus on digital technologies as promoted in the project.

The subsequent sections report on a first study that was performed in summer 2017 within the context of the project. For the study, we have been interested in older people with little to no prior experience using interactive digital media. The design included playing three different games on a tablet. In this context, we have been interested primarily in the following research question:

\section{- How far can game-playing facilitate the encouragement of digital adoption by elderly people?}

This concerns the engagement of elderly individuals in game-playing in the context of digital systems. Our goal was to find out whether and how older adults can be motivated to interact with digital artefacts, in particular touchscreen technologies, through playing games. Game-playing could create a positive attitude towards adopting digital media for personal use, in particular when individuals lack experience of interacting with these technologies [19]. Using the term "Game Play", we follow Ke's approach and use it "to describe the overall experience of playing the game", keeping in mind that the term is used to describe "what [a] player does" [19].

Empirical findings indicate the importance of facilitation when learning through game-playing. Hence, we finally added a research question corresponding to the facilitation of digital capacity building in elderly learners:

- What measures can support digital adoption by elderly people when playing games on tablets?
The more knowledge gained about effective facilitating interventions in the course of skill development and capacity building, the more effectively game-playing can be applied to qualifying elderly people as users of novel interactive technologies. Putting this question further, gameplaying and the subsequent acquisition of digital skills could result in self-managed learning processes [20], including the informed selection and use of games by elderly people.

The paper is structured as follows: In Sect. 2, we provide an overview of the theoretical background of our research activities. The Unified Theory of Acceptance and Use of Technology (UTAUT2) has been applied to design our study, as it subsumes relevant factors in a structured way when encouraging elderly people to engage in digital adoption processes. Furthermore, it enables us to justify the conceptual framework potentially derived from our findings. This framework describes the scope of the study. It also contains fundamental entities, such as experienced problems and, most importantly, it represents essential relationships between these entities (e.g. what kind of interventions could be set to help learners overcome problematic situations). Finally, the framework allows condensed reporting of behaviour occurrences when implementing it in field settings (as we did).

In Sect. 3, we present the methodology of the study before we detail the results in Sect. 4. Section 5 provides the discussion of the empirical results, and Sect. 6 concludes the paper by summarising the findings and describing our future work.

\section{Theoretical background}

This section presents the theoretical background of our research activities. First, we explain the importance of encouraging digital adoption for elderly people. We continue by introducing technology acceptance models detailing the UTAUT2 aspects for helping older people to deal with digital technologies. After explaining the rationale for a game-play approach supporting the implementation of these aspects, we summarise the results for deriving our research approach.

\subsection{Digital adoption by elderly people}

Digital technologies play an increasing role not only in younger adults' daily lives [6], as such technologies are "ubiquitous in everyday life", as an increasing number of services and information is "becoming exclusively accessible via the internet" [6]. Despite this growing importance in daily life, a large number of people are not familiar with the use of digital devices. In particular, older citizens are more often "digitally excluded" as a result of lacking access to the Internet [6]. Only 55\% of individuals aged 65 or older 
are internet users, according to investigations reporting on developments in some European countries [4, 21, 22], while around $80 \%$ of the entire European population uses the internet [23]. While the number of older people who regularly use the internet has increased over the last years [4], there is still significant scope to encourage other older people to become regular internet users.

Investigations reveal that digital technologies can facilitate daily activities, strengthen social contacts, and reduce loneliness. Digital media thus have the ability to facilitate social inclusion and empower elderly people [24]. A telephone survey with 1000 people aged 65 and older in Switzerland revealed that individuals with "a high affinity of technology will presumably use the Internet to cope with everyday life" more than others [4]. Due to the fact that a considerable part of the older population is still not familiar with digital technologies, it becomes necessary to look for relevant aspects to help and encourage this particular user group to adopt digital practices and competencies [25].

\subsection{Technology acceptance and use}

Different models describe relevant aspects that influence the use and acceptance of technology, and one of the most popular is the technology acceptance model (TAM) [26]. In the original version, Davis describes two predictors-perceived usefulness and perceived ease of use - that influence the use of technology [27]. As a result, two advanced versions of TAM-TAM 2 and TAM 2'-deal with determinants for each of these predictors, respectively, and a further version, TAM 3, integrates them [26]. Although TAM "was the leading model" for explaining technology acceptance, different models deal with this issue also [26], with Venkatesh et al. [28] analysing eight different models: (1) the theory of reasoned action; (2) the technology acceptance model; (3) the motivational model; (4) the theory of planned behaviour; (5) a model combining the technology acceptance model and the theory of planned behaviour; (6) the model of PC utilisation; (7) the innovation diffusion theory, and (8) social cognitive theory. With their analysis, Venkatesh et al. developed the UTAUT, a unified theory that "integrates elements across the eight models" [28], which was "originally developed to explain employee technology acceptance and use". The modified version, UTAUT2, focuses on consumer use [17].

We use UTAUT2 as a framework for this contribution due to our interest in the digital adoption of older people as consumers. Venkatesh et al. [17] describe seven determinants that play a significant role in "consumer technology acceptance and use":

- Social influence describes how far "consumers perceive that important others (e.g. family and friends) believe they should use a particular technology" [17];
- Performance expectancy describes how far people believe that using the system will improve their work performance [17];

- Effort expectancy means "the degree of ease associated with consumers' use of technology" [17];

- Facilitating conditions determine how far somebody perceives support as adequate when using a new system [28];

- Hedonic motivation describes the degree of fun when dealing with technology [29];

- Price value refers to the "cost issue of technology use in the consumer setting" [28];

- Habit is defined as "a perceptual construct that reflects the results of prior experiences" [28].

\subsection{Technology acceptance and use in the elderly population}

Concerning our primary research question, we focus on the particularities for older adults with little to no prior experience using digital technologies. The importance of age and experience on digital adoption is also evident in the research of Venkatesh et al. [17], who describe gender, age, and experience as key moderators in UTAUT2. Furthermore, some researchers examine the particularities of older citizens when using digital technologies. For example, Zhou et al. [30] stress that "older adult's acceptance of handheld computers differs from general population's acceptance" and thus needs to be examined explicitly. Macedo [10] used UTAUT2 to investigate the "acceptance and use of information and communication technology by older adults", and discovered that most UTAUT2 determinants are "relevant for this population group". Performance expectancy and facilitation conditions were the most important predictors. Furthermore, effort expectancy, social influence, hedonic motivation, and habit were also significant in this investigation. Price value was not a significant predictor in Macedo's study [10].

Other empirical research findings about elderly people and their use of technology are related to determinants of UTAUT2. Hence, we could identify inputs relevant for encouraging elderly individuals to adopt digital practices. Consequently, we focus on those determinants of UTAUT2 and those results of related studies that can be influenced by our research activities.

Due to the project's setup, we do not consider habit and price value any further. Since we were able to provide the digital devices (i.e. tablet computers) in our research settings, participants who did not possess devices personally could join the study. Therefore, the price value of the device is not relevant for the participants in the study. Moreover, the participants were selected according to their level of competence, meaning that they should have little to no prior 
experience with interactive digital media. Hence, habit was not taken into account, as it is defined as "a perceptual construct that reflects the results of prior experiences" [28].

Finally, UTAUT2 facilitates our research design with respect to the following aspects:

- Social influence Investigations reveal that a lot of elderly people in possession of digital devices do not buy their first digital mobile phone by themselves. Instead, it is "often given to them or bought for them" [31]. A literature review conducted by Zhou et al. [30] shows younger people's influence on older people when using digital devices, which "is not always positive". Providing elderly people with functions identical to those embraced by younger people could be too difficult for first-time elderly users, and "this bad impression may influence their upgrade behavior" [30]. These findings indicate the need to take into account the specific needs and behaviours of elderly individuals when aiming to include them in the digital world.

- Performance expectancy Arning and Ziefle [16] found that older adults consider the "usefulness of a device" in the course of evaluating its ease of use. Therefore, it may be helpful to be aware of the practical significance of the devices. Mallenius et al. [32] came to comparable results in their study, in which they found that the use of mobile devices has to "deliver a real value" for elderly people.

- Effort expectancy Arning and Ziefle [16] found that effectiveness is more important than efficiency when evaluating the perceived ease of use for elderly people in comparison with younger individuals. Hence, the "easy and effort-free" interaction with digital devices is more crucial for younger people than for older ones. In contrast, the successful use of digital devices is more essential for older adults than for younger ones. However, investigations have already found usability aspects that can improve handling interactive media for elderly individuals. For example, a device with a very small screen is not as easy to handle or view for elderly people as a device with a bigger screen [30]. Hence, we can assume that the required perceived success in completing a task can still be facilitated by devices that are easy to use.

- Facilitating conditions Barnard et al. [6] show that the "availability of support, technical and emotional, is crucial", and "also influence how people experience difficulties". Furthermore, Arning and Ziefle [16] argue that "constructive feedback regarding the tasks' success might reduce an older adult's hesitancy and lack of confidence when interacting with technical devices".

- Hedonic motivation Some research has focused on digital game-based learning [33] due to the positive effect of playing games on motivating individuals to learn. Wang et al. [34] stress that a positive effect of playing digital games is getting players engaged and motivated. Additionally, playing games can be fun, and fun promotes learning [35].

\subsection{Gaming and learning}

A literature review on gaming by Connolly et al. [36] shows that different academic papers deal with the benefits of playing digital games, and that game-based learning "is being used across many curricular areas, most notably in health, business and social issues". The interest in games in a learning context has, on the one hand, motivational reasons. Games seem to be "motivating and enjoyable" for the players. On the other hand, playing games can coincide with learning. The literature review indicates different learning outcomes that can be assigned to the following categories: knowledge acquisition, perceptual and cognitive, behavioural, affective, motivational, physiological and social outcomes.

Given the positive effects of gaming, it is not surprising that older adults seem to have a "great potential to accept" digital game-based learning [13]. In general, around $11 \%$ of American people older than 50 play digital games, which makes this group relevant for the gaming industry [37]. In addition, the health sector has recognised the potential of games. Exergaming is one example of combining digital games with "physical exertion or movements that are more than sedentary activities and also include strength, balance, and flexibility activities" [38]. Older adults are motivated to play exergames [39]. In particular, the elements of the play make the "exercise program" attractive [40].

However, it is not just games designed for particular purposes that can affect the lives of older people positively. Kaufman et al. [14] describe motivational aspects and other additional benefits for older adults who play digital games, including "mental experience", "dealing with loneliness or depression" or "developing confidence". Furthermore, the findings - based on a survey of 463 older people who play games on digital devices-indicate that playing games can adhere to acquiring digital competencies. The majority of the participants reported an increase in cognitive, computer, and internet skills. Wang et al. [13] came to comparable results in their study with older computer users. Since playing digital games "only requires the most fundamental computer skills" [34], learners can start playing at a low competence level and can "gradually accumulate computer skills while improving their gaming skills". The participants of the study also believed that playing computer games helps to improve their computer skills [13]. It needs to be noted that the described effects of digital games on computer or internet skills are based on self-reporting of older people who play these games. These studies did not examine the effects of digital games on digital skills beyond self-reporting. 
However, the results justify following a game-play approach in further investigations.

\subsection{Summary}

After providing an overview of findings that influence the use of technology by older people, we summarise our results in order to substantiate our research approach:

- It seems important for elderly individuals that their social environment communicates the significance of using digital devices. Elderly people have to be aware of the value of digital media in order to accept and use it;

- It is helpful to observe usability aspects when choosing a device for digital adoption;

- Additionally, experiencing success in a task accomplishment, as well as enjoyment when using digital technologies, has a positive influence on future use and ultimately technology acceptance. Game-playing seems to be particularly relevant when attempting to provide fun experiences;

- Guiding the process of technology adoption is particularly crucial for elderly people who are exterior to the digital world. Facilitation needs to be provided for orientation and situation-sensitive help.

Analysing the various factors leads also to the finding that the last aspect, "facilitation", cannot be viewed in isolation from the other aspects [41, 42]. As described above, the "availability of support" and "constructive feedback" are essential for novices. Still, several interventions are part of constructive "facilitation": providing positive social influence, making people aware of the value of digital media, providing devices that are easy to use, and encouraging fun experiences. Thus, we consider "facilitation" to be an essential component in each of the described aspects when helping older people to increase digital adoption [20]. We can hypothesise that encouraging elderly individuals to play digital games on touchscreen technologies combined with offering support through a facilitator can be an appropriate approach for them when adopting digital media [25].

\subsection{Research approach}

In the following we discuss the approach of this study concerning the described aspects based on UTAUT2. In this context, it is important to highlight that we do not explicitly investigate UTAUT2 in our study. Rather, we use the findings concerning this model for developing and justifying our research approach: encouraging people to play digital games corresponds to the aspect of social influence. We consider the performance expectancy and effort expectancy aspects with the choice of a tablet as a device for playing digital games. First, from smartphones to ticket machines, the number of different media devices with touchscreens has increased over the last years. Due to the need to use touchscreens in everyday life, playing digital games should include training individuals to handle digital devices and to learn the necessary gestures such devices require. Second, the size of the screen is a relevant usability aspect, especially for older people. Although there is no clear "conclusion about which display size is best", investigations show that older adults' preference of 7 or 9 in. is "[1.5] times as strong as [...] 5 inches" [30]. Thus, tablets instead of smartphones could facilitate the ease of use because of the presence of a larger screen, which can promote successful experiences. As argued above, experiencing success in task completion when using digital technologies is essential for elderly people when using it for the first time. Furthermore, we want to promote successful experiences by providing games that are easy to accomplish. Using game-playing as an approach should encourage fun and thus hedonic motivation. Additionally, a facilitator should accompany the gamers to provide technical and emotional support in order to ensure successful gaming. Therefore, the facilitation conditions aspect needs to be considered.

After a literature review that led us to follow a facilitated game-play approach to encourage digital adoption by elderly people, the following section discusses the methodology for examining this approach.

\section{Methodology}

This section describes the methodology of the study at JKU in the context of the project GIRDA, which was conducted with recognition of ethical guidelines according to the principles of empirical research [43]. Being interested in how far game-playing can facilitate encouraging digital adoption for elderly people (see also Sect. 1), we hypothesise that playing digital games helps elderly individuals to experience digital media in a playful way, which finally leads to the successful adoption of digital technologies. However, the findings from existing theories allow us to conclude that facilitation seems to be crucial when people learn to operate new technologies. Therefore, we have added the following research question: What measures can support digital adoption for elderly people playing digital games on tablets?

Studying the adequacy of interventions helps to reflect on the methodological setting and simultaneously improve the facilitating conditions that are relevant to the adoption of novel digital technologies. The first issue allows for the improvement of the setting of future studies in this field, whereas the second one enables us to develop a guide for facilitators when elderly individuals play games in digital 
adoption settings. The latter is actually a subsequent work package in the GIRDA project.

\subsection{Data collection}

For generating the necessary data, we approached older people with little or no prior experience using digital technology. The Association for Elderlies in Upper Austria (Oberösterreichischer Seniorenbund) is a partner organisation in the project, and represents a large number of active elderly members. As such, we published a call for participants in the association's magazine, specifically requesting senior citizens with little or no prior experience with touch screens. This led to a final selection of 20 members who reported to have little or no digital experience. This cohort included 15 women and 5 men, all of whom were aged between 57 and 87 years. Seven pairs and six individuals came to our office during the summer period in 2017. Each of the participants provided informed consent to contribute to our research and for the respective processing of data for anonymised presentation. Choosing a design with pairwise and single players allowed us to recognise differences in support measures for collaborative and single players.

In order to practise handling touchscreen-based devices, the participants were requested to play three different games on a tablet with a 9.7 in. screen. They started by drawing a picture (app: Paper), followed by completing a puzzle (app: Puzzle Man Pro), and finished the session by playing the game "Candy Crush". The first challenge, "drawing", was selected to introduce basic drag and tap actions and the basic usage of a tablet. The elderly people were asked to draw figures of their own design for around $15 \mathrm{~min}$. Pairs were required to draw a single figure collaboratively. The participants were encouraged to practise the "dragging" gesture using the apps "Puzzle Man Pro" and "Candy Crush".

At the beginning of each session, a team of two researchers introduced the setting and the game, in order to ensure that the learners were aware of the tasks to be accomplished. When explaining the usage of the tablet and the rules of the game, we demonstrated the gestures they needed to play. For instance, for the app "Paper" we illustrated how to select a pen or colour from the toolbar and how to draw with it. After providing this information, we encouraged them to ask us questions in case they anticipated problems. Then, the participants played autonomously (pairs played together, single learners played alone) to get the chance to make their own decisions and have their own experiences when using a tablet. Once the participants asked questions in the course of playing, the researchers took the role of facilitators.

We made two videos per session in order to record data. The first video focussed on the tablet and how to interact with it using fingers, whereas the second one captured body movements in order to be able to analyse body language.
Besides the video recording, we compiled fieldnotes for comprehensive documentation of each session. The main interest in the video data is to find out about the support measures when elderly people play games on tablets for the first time and need to be encouraged for digital adoption.

We additionally conducted interviews with the learners after each game. The interviews contained closed questions that were complemented with open questions, allowing unstructured feedback and verbal articulation of experiences. Our key interest was to find out about the attitudes of the elderly persons towards digital devices after playing games on the tablet (for detailed items, please see Sect. 3.2). We also made video recordings to document each interview.

To examine the effects of playing digital games on tablets for digital adoption, it was necessary to contact the participants again after some time. We prepared a questionnaire with open questions for structuring the planned telephone interview. Each participant was asked to describe any changes they may have experienced when dealing with touchscreen-based devices since the game-playing session in our office. The interviews were held approximately 3 months after the initial game-playing sessions.

Having described the method of recruiting participants and collecting the data, in the next section we report on the collected data and the analysis focusing on the research questions of that study.

\subsection{Data analysis}

This section reports on our qualitative use of semi-structured interviews and video recordings. Even though the focus is on qualitative data analyses, we provide some quantitative data based on interviews with three closed question. The findings with respect to these closed questions should give a first impression of how much fun the participants had when playing. In-depth analyses are made based on the semi-structured interviews and the video clips. For analysing the semi-structured interviews, we clustered the answers according to common topics, summarising and comparing them. The analysis of the video clips is based on a "thematic analysis" [44]. This section describes the data basis and the data analyses in detail, separately for each research question.

How far can game-playing facilitate encouraging digital adoption for elderly people?

As we learned from the theoretical background given in Sect. 2, having success and fun can promote digital adoption, especially for older people. Based on this finding, our research setting was designed for elderly individuals to combine having success and fun while playing digital games that encourage digital adoption. Therefore, on the one hand we were interested in the feelings and 
attitudes of the participants after playing each game, whereas on the other hand, we focused our research activities on the effects of playing digital games on digital adoption. Accordingly, we conducted interviews at different times: after playing each game, and several weeks later. After playing each of the games, each participant received a questionnaire with the following items (initial measurement):

- I enjoyed playing the game;

- I would be interested in playing more games;

- I feel more positive about digital systems (e.g. tablets, laptops, iPads, etc.) after playing this game.

The items were rated on a five-point Likert scale and were designed to inform about the feelings and attitudes the elderly people possessed towards adoption based on their first experience of playing digital games on touchscreenbased devices. The answer options were "strongly agree", "agree", "neutral", "disagree", and "strongly disagree". One methodological advantage of closed questions concerns the comparability of answers across the set of participants [15]. Accordingly, the Likert scale allows us to provide a clearly structured overview of the selected options of the participants. The aim of this questionnaire was to get a first impression about their attitude and feelings directly after playing. For analysing the ordinal scale, we have calculated the frequencies for each item. The result shows the distribution of the answers. In addition, we asked the participants to elaborate on their answers in an open discussion in order to receive more detailed information.

Secondly, to examine the effects of playing digital games, we contacted each participant several weeks after the gaming session. The aim of this was to ascertain whether they experienced any behaviour changes in relation to touchscreen devices since participating in the games. We opted for a telephone interview based on a semi-structured questionnaire with open questions. The telephone interview was deemed sensitive to the physical conditions of some of the participants and the distance between their homes and our research centre, which could make personal arrangements more difficult. Therefore, it was anticipated that the response rate would be higher when contacting individuals via telephone instead of trying to meet face-to-face. Open questions give participants the opportunity to describe their attitudes and experiences in their own words [45]. Hence, it was possible to compile a detailed report about the usage of touchscreenbased devices since study participation for all participants. The following list gives an overview of the key questions of the semi-structured telephone interview:

- How has your usage of tablets and smartphones changed since participation in the study?
- When using ticket machines at the train or tram station, it is often necessary to operate a touchscreen. Have you used such machines since participating in the study? If so, do you think you are able to use such machines more quickly than before you played digital games on a tablet in the project?

- Which help have you received for using digital media since participating in the study?

To analyse the changes of behaviour with respect to using digital media, we clustered the answers according to common topics, comparing and summarising them in order to identify the effects of tablet-based game-playing on digital adoption.

What measures can support digital adoption for elderly people playing games on tablets?

For examining the support measures when elderly people play digital games for the first time on touchscreen-based devices, we analysed the video recordings of the session. We merged the two videos of each session to enable a simultaneous observation of gestures and body language. The analysis was guided by a thematic analysis. This method is "independent of theory epistemology" and "provides a flexible and useful research tool, which can potentially provide a rich and detailed [...] account of data" [44].

We are interested in discovering learner needs when handling a touchscreen-based device in the game-play approach without focusing on a theoretical background. This approach allows an inductive way of analysing the data [44]. It is appropriate for our research question, as Braun and Clark [44] explain that inductive thematic analysis is "data-driven" and is "therefore a process of coding the data without trying to fit it into a pre-existing coding frame". Consequently, the analysis of the video clips was driven by the research question.

Braun and Clark [44] suggest "familiarizing yourself with your data $[\ldots]$ before you begin your coding, as ideas and identification of possible patterns will be shaped" as the first step in a thematic analysis. Accordingly, we obtained an overview of the data before we started the coding. In the process, it turned out that support was always linked with the participants experiencing a problem when acting with the tablet. As such, analysing the support must coincide with analysing the problems that occur when playing games on the tablet. Finally, we could identify the following set of questions that had been guiding the coding and analysis of the data:

- What were the problems while the participants were playing games on the tablet?

- How did the participants react once a problem occurred? 
- What support was given?

- Who provided the support?

- What happened after providing support?

After becoming familiar with the data, the initial codes have to be generated [44]. "Codes identify a feature of the data (semantic content or latent) that appears interesting to the analyst" [44]. As follow-up steps of a thematic analysis, Braun and Clark [44] suggest "sorting the different codes into potential themes" followed by "reviewing [...] defining and naming themes" before a report can be produced. Thus, we have coded and analysed the video data accordingly. The identified themes are shown in Sect. 4.2.

Having described our data basis and analysis, the findings for answering our research questions are presented in the following section.

\section{Results}

This section presents the findings of our study. We report the results separately for each research question. We first describe how far game-playing can facilitate encouraging digital adoption for elderly people. We then present our findings with respect to the support measures in these learning situations.

\subsection{Encouraging digital adoption through a gameplay-based approach}

In summer 2017, we organised sessions at the university campus where 20 participants played 3 different games on a tablet, either in pairs or alone. Three months later, we conducted a telephone interview with each participant. As already argued, having success and fun should promote learning. Therefore, we present the results of the participants' feelings and attitudes towards the playing sessions upfront, before moving on to explain how far game-playing facilitated the encouragement of digital adoption.

The interviews conducted after the game-playing session led to conversations with the participants about issues that were not part of the questionnaire. These additions were interesting for our research activities, as the participants reported that losing track of digital media can coincide with feelings of social exclusion. On the one hand, the majority of the participants mentioned that digital media plays a significant role in the lives of their younger relatives. When having family meetings, children and grandchildren use their smartphones and talk about WhatsApp or Facebook, while the older generation often do not understand what their family members are talking about. On the other hand, a large number of public services require individuals to be able to deal with digital

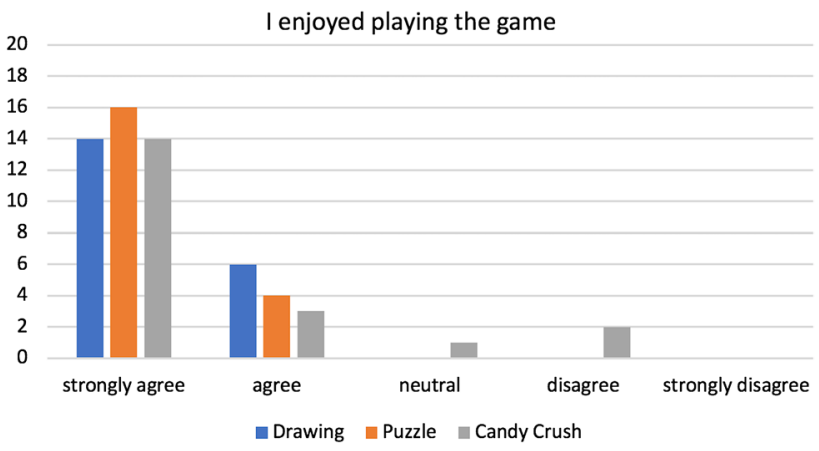

Fig. 1 How enjoyable the games were to play

devices, and some of the participants complained about the "complicated ticket machines" at railway or tram stations. It was often hard for them to buy a ticket and sometimes they needed help from people passing by. Less than half of the participants remarked that they had no problems with buying tickets for the tram or the railway. Furthermore, one woman reported that she had been a member of an organisation for years, but that recently it had only become possible for her to receive the regularly published newsletter via email. This made her angry because she did not have Internet access and thus was excluded.

In summary, all participants perceived digital devices as important media in everyday life. As described in more detail below, less than half of the participants possessed their own touchscreen-based device before the study. However, all of them indicated that they would be interested in learning to deal with smartphones or tablets because of their everincreasing importance in our society. The majority admitted that they did not dare to deal with such media due to specific reasons. Some of them were worried about breaking something when using it or thought that they were too old and clumsy to learn how to use the device. Others complained about the lack of support.

Nevertheless, these 20 elderly people were interested in participating in the study. As mentioned earlier, they completed three different games on a tablet. Figure 1 illustrates how enjoyable the playing sessions were perceived to be by the participants, while Fig. 2 shows the levels of interest in playing more games. The survey was made immediately after each game.

Figure 1 shows that nearly all of the participants enjoyed playing the digital games. Only two people did not have fun while playing Candy Crush, while one other person was neutral to that game. It turned out during the interviews that the puzzles and drawing challenges were more popular than Candy Crush amongst the participants. The analysis of the conversations and the video clips indicate that some people did not fully understand the rules of Candy Crush, which could be one reason for this finding. 


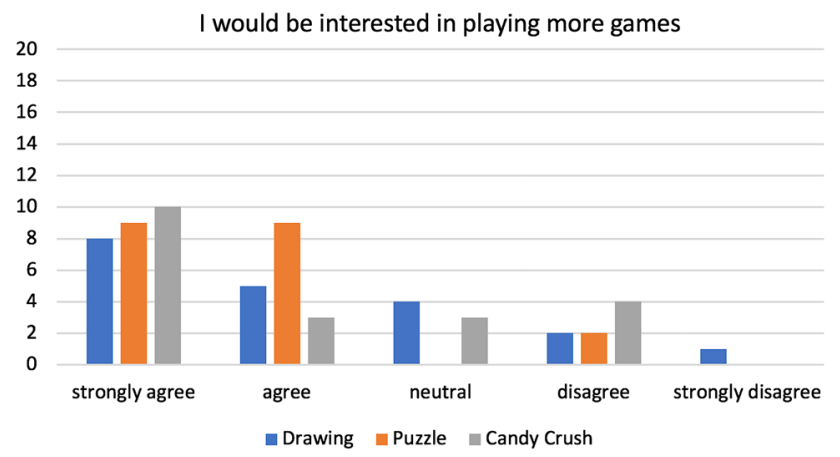

Fig. 2 Interest in playing more digital games

As Fig. 2 illustrates, the majority of the participants are interested in playing more games, now that they have experience of completing a game. A comparison of the two figures shows that the majority strongly agreed with the statement "I enjoyed playing the game", though fewer participants strongly agreed with the statement about being interested in playing more games in the future. The conversation with the participants provided a potential reason for this finding. Participants mentioned that they enjoyed playing the digital games a lot, but some of them would be more interested in learning to accomplish other tasks using the tablet (e.g. making photos or "googling"). Hence, the interest in playing more games was not particularly high for some of them.

Overall, the feedback was very positive. The reaction to the next statement of the questionnaire shows surprising results. The participants had to respond to the statement "I feel more positive about digital systems after playing this game", using a Likert scale. Many of them said they could not give a correct answer because they never had a negative attitude towards digital systems. They were rather shy and afraid of learning digital technologies, though their basic feelings about digital systems had been and remained positive.

In summary, playing digital games on a tablet device seems to have been a positive experience for the elderly individuals who participated in the first study. However, our main interest was to find out to what extent game-playing could facilitate encouraging digital adoption in elderly people. Therefore, we had to examine their behaviour changes using touchscreen-based devices after participating in the game-playing sessions. As described in Sect. 3 in detail, we could conduct semi-structured telephone interviews with all participants 3 months after participating in the study. Section 3.2 gives an overview of key questions and the method utilised for analysing the responses. In the following, we summarise the changes observed by participants in relation to using digital devices, as reported in the course of the telephone interviews. Six out of 20 participants owned their own tablet or smartphone though had little experience using it before their participation in the study. Around 3 months later, this number had doubled. All in all, 12 participants told us in the telephone interview that they used their own touchscreen-based device regularly. One participant explained that he did not want to own a device, but that he would borrow one from his children to use it from time to time. Furthermore, 16 of the 20 participants stated that their handling of digital devices had changed in a positive way since attending the game-playing session. The following part gives an overview of the experienced changes as reported by the participants:

- Four of the six participants who had a smartphone or tablet before attending the study reported more frequent use. The majority of all tablet or smartphone owners stated that they used it daily or even up to several times a day. Only four of them reported a less frequent use of 1-3 times a week;

- When asking the participants what they do with their interactive media, a wide range of practices was mentioned, including email, photo, WhatsApp, Google, calendar, weather, games, news, or Facebook. A lot of them focused on two to three different applications. One 87-year-old woman only did digital puzzles besides or looked at photos on her tablet. Another woman stated that she was able to read messages, to read the news, and to take photos with her smartphone. Only a minority of participants engaged with social networks, Google, WhatsApp, or emails;

- Nearly every user told us that their handling of touchscreen-based devices had improved since the session. They felt more competent, and observed that their handling of the devices was quicker than before attending the game-playing session. They talked about more fun and less fear about getting something wrong when using the devices. The interest in dealing with digital media and learning something new had also increased. One woman found out that handling a tablet or smartphone was not as difficult as she had thought before;

- Four participants talked about changes when using ticket machines (although three of them owned no digital media devices 3 months after the study, either). They were more interested in using such ticket machines and had less fear of buying a ticket. Furthermore, they learned through game-playing to be more patient. All in all, they felt more competent when using digital ticket systems, although some of them complained about the very complicated new system of the ticket machines at the train stations.

Only one out of the five participants who reported no change in using interactive media was not interested in using them anymore. The remaining four of these five participants were thinking about buying a tablet or smartphone soon; 
The majority of the elderly people received help from more experienced relatives when handling digital devices after the game-playing session. Twelve of the 20 participants mentioned that they asked children or grandchildren for help in case they experienced problems. Two of them additionally signed up for a course.

In summary, the majority of the respondents reported significant changes in using interactive media. Furthermore, playing games in the study was fun, as almost all participants emphasised they enjoyed game-playing, and the session was interesting for them.

\subsection{Learning support}

In the previous section, we described how game-playing could encourage digital adoption. Now, we turn to the second research question: Which measures can support elderly people when playing digital games on tablets for encouraging digital adoption? As described in Sect. 3.2 in detail, we conducted a thematic analysis for answering this question. In the following, we present the results of the analysis of the video clips.

According to inductive thematic analysis, the coding of the video clips was driven by the research question of this section. Thus, we coded all observed situations of the playing sessions that were related to problems, support provided, and individual reactions to the problems. Finally, we could identify 11 different themes. The following table describes these themes with exemplary items of the video analysis, clustered into "Problems", "Reactions to the problems by the learners", "Supportive activities", and "Observed outcomes".

Table 1 presents the observed problems and support in the playing sessions. Furthermore, it describes the reactions and outcomes after a problem had occurred. In order to find out which measures can support elderly people when playing digital games on tablets, we had to analyse the support processes as a whole, focussing on the sequence of handling learning difficulties and observed outcomes. Although the video clips were analysed qualitatively, we included a description of the frequencies of the observed sequences. This enrichment allows researchers to visualise which behaviour the learners show after a problem has occurred. Despite the small sample, a representation of the frequencies of the sequences can provide valuable information about the importance of a facilitator, a partner, or the possibility to solve problems without external help. Hence, frequencies in our analysis provide additional valuable data for answering the research question. Figure 3 shows these sequences in a conceptual framework. The framework can be used to represent problems, interventions, and observed outcomes in a context-sensitive way.
Figure 3 reveals such support by showing how the games led to successful tablet interactions a total of 15 times. These interactions are summarised with the term "Progress". Additionally, we could observe 10 situations in which the participants were searching for support from the facilitator directly after a first introduction. Furthermore, problems with tablet interaction follow the introduction in eight cases. All in all, we could identify 56 problem situations when participants were playing the games on the tablet. As described in Table 1 in more detail, we could classify the observed problems along three different themes: "Mistakes in tablet handling" and "Failure to complete motor control" occurred equally often. In contrast, we could identify the theme "Misunderstanding the rules of the game" less often.

The problems led to three different reactions by the participants. In most cases, the elderly people sought help after realising that a problem had occurred. While seeking help from the facilitator occurred 23 times, we could identify only 6 situations in which the participants sought help from their partner. Finally, in 10 cases some of the participants attempted to solve problems on their own. Moreover, problems did not always lead directly to a reaction by the participants. Sometimes the facilitator or the partner realised the problem or reacted to it first. This was usually the reason for supportive activities. We also observed a situation in which a participant did not react to the problem and was still able to continue with the task.

When the participants tried to solve problems by themselves, they had success in five of the ten observed situations. In four other cases, trying it independently led to other problems or to support by the facilitator. We also observed one situation in which the person asked the facilitator for help after an unsuccessful attempt to solve the problem independently.

The facilitator and partners usually provided support after a learner sought help. Depending on the problem, they usually decided to use explaining, demonstrating, or instruction as support methods. Questioning as a method for helping people to continue the task was only used two times by the facilitator. The data show that the given support was not always correct. Both facilitators and partners gave inaccurate explanations or did not act correctly in several situations. Nevertheless, we could observe progress after supportive activities in most cases. Hence, the learners were usually able to continue the task successfully. However, support did not always lead to successful learning outcomes. We could identify 15 instances of re-occurring problems after support by the facilitator and three instances after support by partners. As a result, the learners had to seek help again, or the facilitator and partners reacted directly to the continuation of the problem. When analysing the situations with recurring problems, we could observe several negative attitudes to the 


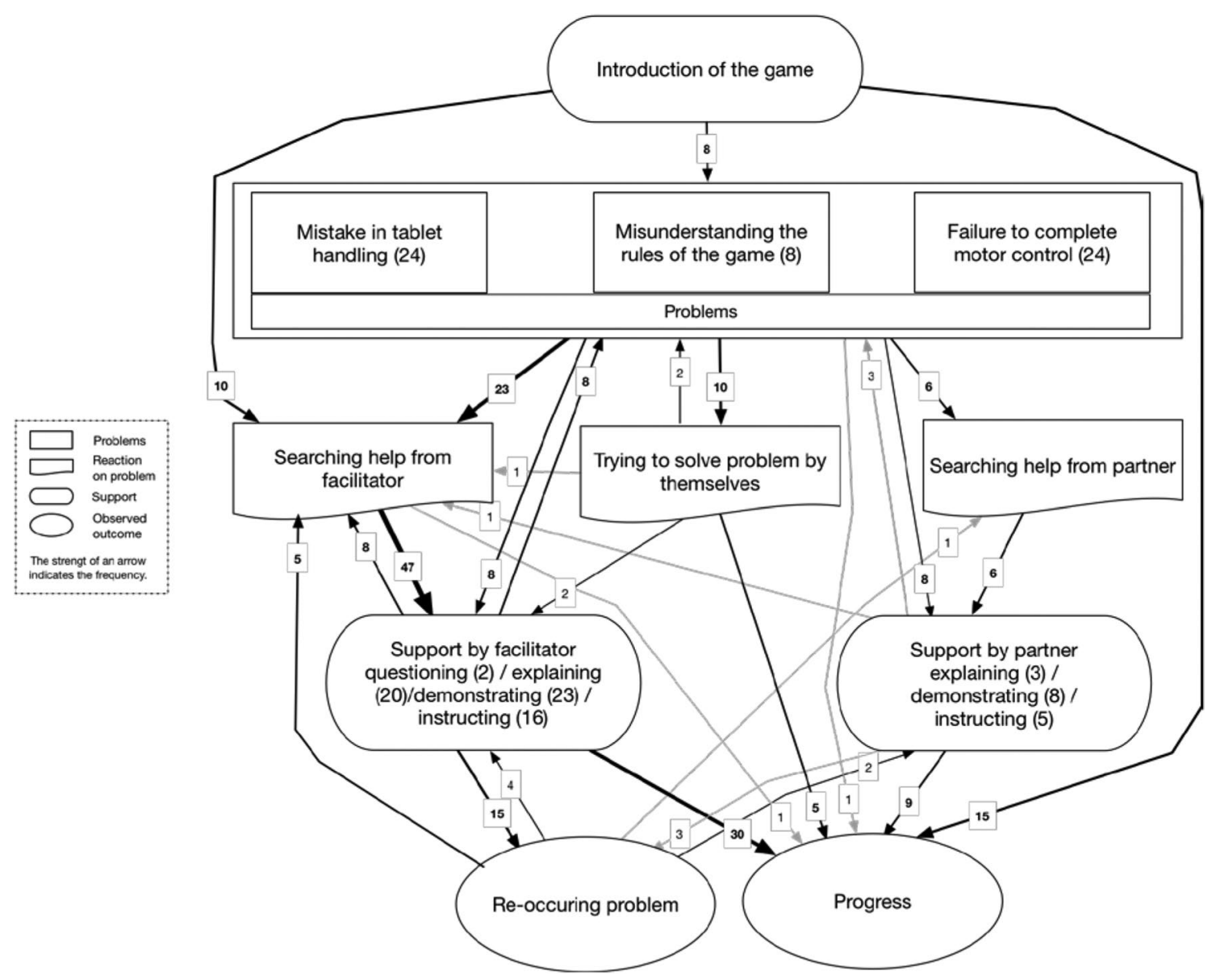

Fig. 3 Sequences of handling learning difficulties and observed outcomes

touchscreen interaction by the participants. Sometimes they got a little nervous or impatient, and some started to sweat or talked about their "clumps" (fingers) as reasons for their problems with tablet interaction.

In summary, the data show that the majority of the problems in tablet interaction led to support by the facilitator. Most of the problems were related to mistakes in tablet handling and failures to complete motor control. The support was usually given through explaining, demonstrating, and instructing. The number of situations with successful learning outcomes after support was twice as large as re-occurring problems. The thickness of the arrows in Fig. 3 illustrates this finding. Nevertheless, supportive activities did not lead to successful learning experiences in all cases. In some cases, recurring problems were accompanied by negative, nervous, and impatient attitudes and behaviour by the participants. We will discuss and interpret these findings in the following section with the theoretical background of this paper.

\section{Discussion}

In the previous section, we have described the participants' feelings and attitudes towards playing games on a tablet in the first study of the Erasmus+ project GIRDA and the effects of the playing sessions on digital adoption. Furthermore, we reported on the support measures in the study. The objectives of this paper are to answer the following two research questions: How far can game-playing facilitate the encouragement of digital adoption in elderly people? and: What measures can support elderly people when playing digital games on tablets for encouraging digital adoption? In this section, we discuss the results with reference to the theoretical background and with a focus on the research questions of this paper.

According to UTAUT2 [17], social influence and performance expectancy are two of the seven determinants of user acceptance and usage behaviour. With respect to 
Table 1 Identified themes

Themes Description and examples

Problems

Failure to complete motor control

Mistake in tablet handling

Misunderstanding the rules of the game

Reactions to the problems by the learners

Seeking help from facilitator

Seeking help from partner

Trying to solve a problem by themselves

Supportive activities

Introduction of the game

Support by facilitator

Support by partner

Observed outcomes

Progress
This is usually triggered by problems with swiping the toolbar or tapping to choose a colour or pen because of inaccurate motor control:

Swiping the toolbar not possible (when toolbar not visible)

Learner tries to swipe but cannot activate the toolbar (App: Paper)

Tapping in the wrong place

Learner wants to choose a colour $\rightarrow$ taps not exactly enough $\rightarrow$ it does not work (App: Paper)

This is triggered by different reasons, which are all related to the handling of the tablet:

Touching the screen at the same time as partner

Learner taps on the screen although the partner is drawing (App: Paper)

Using a wrong gesture

Learner tries to swipe to choose the eraser instead of tapping $\rightarrow$ it does not work (App: Paper)

Choosing wrong icon

Learner wants to open the tool bar but chooses the wrong icon and opens another window (App:

Paper)

Using fingernails for drawing

Learner uses the fingernail for drawing $\rightarrow$ it does not work (App: Paper)

This is triggered by difficulties in understanding the rules of the game:

Learner chooses the wrong candies (App: Candy Crush)

This is usually triggered by a request for help from the facilitator by the participant:

Request regarding the handling of the tablet

Where should I tap to activate the tool bar (App: Paper)?

Request regarding the gesture

How can I move the puzzle pieces (App: Puzzle Man Pro)?

Request regarding the rules of the game

Which directions are possible to move the candies (App: Candy Crush)?

This is usually triggered by a request for help from the partner by the participant:

Where did you choose the colour (App: Paper)?

It doesn't work! ... Look (App: Paper)!

This is triggered when the participant tries to solve a problem by themselves:

Learner tries to close the open window, but it does not work (App: Puzzle Man Pro)

Learner starts to draw without choosing a pen $\rightarrow$ it does not work $\rightarrow$ trying to choose a pen (App:

Paper)

The facilitator explained the rules of the game and the handling of the tablet at the beginning of each task. This usually included a short demonstration of the necessary gestures

This describes different supportive activities by the facilitator:

Support through explaining

Facilitator explains how to move the puzzle pieces (App: Puzzle Man Pro)

Support through demonstrating

Facilitator demonstrates how to open the toolbar (App: Paper)

Support through instructing

First you have to choose a pen and then you can draw (App: Paper)

Support through questioning

Did you choose a pen (App: Paper)?

This describes different supportive activities by the partner:

Support through explaining

It is not possible to write with white on a white paper (App: Paper)

Support through demonstrating

Partner swipes and successfully opens the toolbar after the other partner was failing to open it

(App: Paper)

Support through instructing

Try again to choose a pen (App: Paper)

This describes situations when learners could successfully complete a task after a problem or could go on after support:

Swiping the toolbar successfully after the facilitator has explained how it works (App: Paper)

Learner finds the hidden puzzle pieces (App: Puzzle Man Pro) 
Table 1 (continued)

\begin{tabular}{ll}
\hline Themes & Description and examples \\
\hline Recurring problem & This describes problems in tablet interaction that occurred several times although help was given \\
& Learner can't choose the colour again because of imprecise tapping gesture (App: Paper) \\
Learner chooses the wrong icon again and opens the incorrect window (App: Paper) \\
Learner chooses the wrong candies again (App: Candy Crush)
\end{tabular}

these determinants, the participants in our study reported that digital media played an essential role in their relative's lives and became more and more important in public services. Therefore, the data show an ever-increasing social influence of digital media on the lives of elderly people. The participants in the study also talked about feelings of exclusion from an increasingly digitised society. From the perceived lack of inclusion, it can be concluded that the elderly individuals who took part in the study wanted to learn to handle digital devices in order to feel more included and to be able to deal with digital social services. In summary, it is evident that elderly people are under social pressure to learn to deal with interactive media and they are aware of the potential value of such devices.

As the interviews show, the acceptance of new digital systems is not a problematic issue for the participants. However, there are obstacles when learning how to handle them. Some participants were afraid to make mistakes or to fail when using the devices. Furthermore, they complained about insufficient support from their relatives. As discussed in the theoretical background, social influence does not always result in positive effects, especially when people are overwhelmed in their learning processes. To encourage these learning processes, we examined a facilitated game-playing approach. We choose a tablet for the playing sessions to lower effort expectancy as described in UTAUT2. However, as discussed in the theoretical background, when using digital devices successful task accomplishment is more important for older people than low effort [16].

Successful and fun experiences during game-playing are evident in our study, and Fig. 3 shows that the participants had successful learning experiences when playing digital games on the tablet. Furthermore, the participants reported having a lot of fun and positive feelings in the playing session, which is related to hedonic motivation according to UTAUT2. Additionally, the telephone interviews revealed that the elderly people's usage of touchscreen-based devices had changed positively 3 months after the game-play session: 16 of the 20 participants stated that the handling of digital devices had improved since attending the study. They reported more fun and less fear when using interactive media. Furthermore, the number of elderly individuals with their own tablet or smartphone had doubled. Four participants talked about positive changes when using ticket machines. Only one of the five elderly users who did not experience any change in using interactive media was not interested in using it in the future. These results indicate that successful and fun experiences when playing games on a tablet encourage positive changes in using interactive media.

Figure 3 shows that the participants had a lot of successful experiences due to supportive activities. In cases of problems, participants preferred asking the facilitator or the partner for help instead of trying to solve a problem by themselves. This finding refers to the determinant facilitation conditions of UTAUT2. As Barnard et al. [6] explain, the "availability of support, technical and emotional, is crucial" and "also influence how people experience difficulties". This is evident in our study. Explaining, demonstrating, instructing, or questioning encouraged progress after problems with tablet interaction. However, Fig. 3 also illustrates that not all learning experiences were positive. We observed re-occurring problems when playing the games, combined with negative and nervous attitudes in some cases. Furthermore, we could identify several incorrect support measures in the study. The facilitator seemed to not always realise which problem had occurred because of a physical distance to the learners. This distance hindered the permanent observation of the learners' tablet interactions. Consequently, the facilitator could not always provide correct and necessary support, which could explain the missing progress.

Additionally, Fig. 3 shows that the number of cases with support by a gaming partner is much lower than the number of cases with support by the facilitator. This number is remarkable, as 14 participants played in pairs and only 6 alone. Therefore, the majority of the participants had the chance to ask a partner for help. In summary, we could observe relatively more successful learning experiences when help was provided by the facilitator than by the partner. Therefore, getting help from more experienced people seems to be crucial in cases of problems. The data of the telephone interview also show that more than half of the participants have sought help from younger, more experienced relatives when using digital devices after the study.

Based on these findings, game-playing on tablets seems to be an appropriate approach for encouraging digital adoption by elderly people. The participants had fun and experienced success while playing the games, and reported improved digital skills and more frequent use of interactive media 3 months after the study. A comparison of Figs. 1 and 2 shows that the participants enjoyed playing games 
very much. However, the commitment to playing additional games was not so high across all of the participants. Two participants had hardly any problems while playing the games, and both revealed a desire afterwards to try out other things with the tablet (e.g. making photos or "googling"). This finding leads to the hypothesis that playing is especially helpful for complete beginners. Following successful experiences, the participants wanted to explore features of the device that could be useful in everyday life.

To ensure successful and positive experiences, adequate facilitation measures seem to be crucial. These include observation of learner actions to realise the actual cause of a problem. The data show that support through explaining, demonstrating, instruction, or questioning helps learners to make progress after a problem has occurred with tablet interaction.

\section{Conclusion}

In this paper, we reported on a study with a facilitated gameplaying approach for encouraging elderly people to adopt digital practices. In Sect. 2, we explained that digital technologies are omnipresent in daily life, raising the fact that many older citizens are not as familiar with interactive media as younger ones. To address this problem, we suggested that digital adoption by elderly people could encourage social inclusion and enhance daily activities. We used the UTAUT2 theory to identify aspects influencing digital adoption by elderly individuals. Based on this theory, we discussed why playing digital games on a tablet could be an appropriate approach for encouraging digital adoption. UTAUT2 also allowed us to argue that facilitation in this game-playing approach should be an important aspect of our study. In this context, we were interested in the following two questions: How far can game-playing facilitate the encouragement of digital adoption by elderly people? What measures can support digital adoption by elderly people when playing digital games on tablets?

The study is part of the Erasmus+ project GIRDA and involved 20 participants who played 3 different games on a tablet in pairs or as single gamers. A facilitator introduced the playing sessions and gave support when the learners needed help. Video documentations of the whole sessions and interviews after playing the games form the basis of the data analysis. Additionally, we conducted semi-structured telephone interviews with all participants 3 months after the sessions to find out about the changes in using interactive media. We analysed the video clips in an inductive way according to a thematic analysis. The items of the closed questions were rated on a five-point Likert scale, which allowed us to calculate the frequencies. Finally, we clustered the answers of the open questions according to common topics.

The analysis of the data shows that game-playing on tablets is an appropriate approach for encouraging elderly people to adopt digital practices. The learners had fun and successful experiences when playing the games. The majority of the participants reported positive changes in handling interactive media 3 months after the playing sessions. Our study shows that facilitation processes are crucial for these successful learning experiences. Based on our findings, we assume that the observation of a learner's action when playing games through the facilitator allows for the provision of adequate support in cases of problem. Missing or inadequate support can lead to re-occurring problems that may coincide with negative learning experiences. The data set indicates that elderly people prefer asking the facilitator for help instead of a partner.

The present study has certain limitations. For example, we suggested that game-playing on tablets is an appropriate approach for encouraging elderly people to participate in digital practices; however, our study does not compare the game-playing approach with other learning approaches. Furthermore, our data indicate that giving support in case of problems with tablet interactions encourages successful learning experiences. The setting of our study, however, did not allow for a comparison with game-playing sessions with or without other support measures. Moreover, the elderly individuals participating in the study were recruited as volunteers through an open call for participation. This might have introduced a bias with regard to technology acceptance, as these people might have had a higher willingness to learn to deal with interactive media than average within their age group. Therefore, the external validity of our study is limited and requires further research in a larger scale study to lead to generally valid results.

Nevertheless, the reported results of this study justify the consideration of game-playing and facilitation as an appropriate approach for encouraging elderly people in digital adoption. Both the feedback from the participants about their feelings after game-playing and their reports about changes in digital skills justify this finding.

In future work, we will further elaborate on game-playing as an approach for encouraging digital adoption. We aim to focus on potential support measures and compare the observable effects to those reported for other learning approaches. Furthermore, we will broaden the scope of the study to address people with low acceptance of digital technologies.

Acknowledgements Open access funding provided by Johannes Kepler University Linz. This research was partially funded by the European Union's Erasmus+ Programme Grant no. 2016-1-UK01KA204-024508. The authors would like to thank Julia Marek for her contribution to the preparation and subsequent conducting of the study. 
Furthermore, we would like to thank Rebecca Wernig and Sarah Zeiml for their contributions to the coding activities necessary to perform the present study.

Open Access This article is distributed under the terms of the Creative Commons Attribution 4.0 International License (http://creativeco mmons.org/licenses/by/4.0/), which permits unrestricted use, distribution, and reproduction in any medium, provided you give appropriate credit to the original author(s) and the source, provide a link to the Creative Commons license, and indicate if changes were made.

\section{References}

1. Schulz, R., Wahl, H.-W., Matthews, J.T., De Vito Dabbs, A., Beach, S.R., Czaja, S.J.: Advancing the aging and technology agenda in gerontology. Gerontologist 55, 724-734 (2015). https ://doi.org/10.1093/geront/gnu071

2. Stone, M.E., Lin, J., Dannefer, D., Kelley-Moore, J.A.: The continued eclipse of heterogeneity in gerontological research. J. Gerontol. 72, 162-167 (2017). https://doi.org/10.1093/geronb/gbv06 8

3. Loos, E.: Senior citizens: digital immigrants in their own country? Obs. (OBS*) J. 6(1), 1-23 (2012)

4. Seifert, A., Schelling, H.R.: Alt und offline? Z Gerontol Geriatr. 49, 619-625 (2016). https://doi.org/10.1007/s00391-015-0965-1

5. Lenhart, A., Horrigan, J.B.: Re-visualizing the digital divide as a digital spectrum. IT Soc. 1(5), 23-39 (2003)

6. Barnard, Y., Bradley, M.D., Hodgson, F., Lloyd, A.D.: Learning to use new technologies by older adults: perceived difficulties, experimentation behaviour and usability. Comput. Hum. Behav. 29, 1715-1724 (2013). https://doi.org/10.1016/j.chb.2013.02.006

7. Chiu, C.-J., Liu, C.-W.: Understanding older adult's technology adoption and withdrawal for elderly care and education: mixed method analysis from national survey. J Med Internet Res. https ://doi.org/10.2196/jmir.7401 (2017)

8. Wortley, D., An, J.-Y., Heshmati, A.: Tackling the challenge of the aging society: detecting and preventing cognitive and physical decline through games and consumer technologies. Healthc. Inform. Res. 23, 87-93 (2017). https://doi.org/10.4258/ hir.2017.23.2.87

9. Raemdonck, I., Beausaert, S., Fröhlich, D., Kochoian, N., Meurant, C.: Aging workers' learning and employability. In: Bal, P., Kooij, DTAM., Rousseau, DM. (eds.) Aging workers and the employee-employer relationship, pp. 163-184. Springer, Cham (2015)

10. Macedo, I.M.: Predicting the acceptance and use of information and communication technology by older adults: an empirical examination of the revised UTAUT2. Comput. Hum. Behav. 75, 935-948 (2017). https://doi.org/10.1016/j.chb.2017.06.013

11. Merriam, S.B., Kee, Y.: Promoting community wellbeing: the case for lifelong learning for older adults. Adult Educ. Q. 64, 128-144 (2014). https://doi.org/10.1177/0741713613513633

12. Brooks, A.L.: Zoom: a serious games intervention design modelwhen games alone are not enough! In: 2016 8th International Conference on Games and Virtual Worlds for Serious Applications (VS-GAMES). pp. 1-6 (2016)

13. Wang, F., Lockee, B.B., Burton, J.K.: Computer game-based learning: perceptions and experiences of senior Chinese adults. J. Educ. Technol. Syst. 40, 45-58 (2011). https://doi.org/10.2190/ ET.40.1.e

14. Kaufman, D., Sauvé, L., Renaud, L., Duplàa, E.: (2014) Benefits and barriers of older adults' digital gameplay. In: Proceedings of the 6th International Conference on Computer Supported Education, vol. 1. SCITEPRESS-Science and Technology Publications, Lda, Portugal, pp. 213-219

15. Nulkar, G.: Strategic tangle: a cocreated strategy game for management students. Manag. Teach. Rev. 1, 120-127 (2016). https ://doi.org/10.1177/2379298116631111

16. Arning, K., Ziefle, M.: Understanding age differences in PDA acceptance and performance. Comput. Hum. Behav. 23, 29042927 (2007). https://doi.org/10.1016/j.chb.2006.06.005

17. Venkatesh, V., Thong, J.Y.L., Xu, X.: Consumer acceptance and use of information technology: extending the unified theory of acceptance and use of technology. MIS Q. 36, 157-178 (2012)

18. Renaud, K., van Biljon, J.: Predicting technology acceptance and adoption by the elderly: a qualitative study. In: Proceedings of the 2008 Annual Research Conference of the South African Institute of Computer Scientists and Information Technologists on IT Research in Developing Countries: Riding the Wave of Technology. ACM, New York, pp. 210-219 (2008)

19. Ke, F.: (2009) A qualitative meta-analysis of computer games as learning tools. In: Handbook of Research on Effective Electronic Gaming in Education, pp. 1-32. https://doi.org/10.4018/978-159904-808-6.ch001

20. Seabrook, H.J.: Informal learning using tablet computers and apps: a multi-method study of older adults self-managing diabetes. Thesis, University of Calgary (2016)

21. Statistik Austria IKT-Einsatz in Haushalten. https://www.stati stik.at/web_de/statistiken/energie_umwelt_innovation_mobilitaet /informationsgesellschaft/ikt-einsatz_in_haushalten/index.html. (2017). Accessed 19 June 2018

22. Statistisches Bundesamt: Staat \& Gesellschaft - IT-Nutzung Statistisches Bundesamt (Destatis). https://www.destatis.de/DE/ ZahlenFakten/GesellschaftStaat/EinkommenKonsumLebensbedi ngungen/ITNutzung/ITNutzung.html. Accessed 19 June 2018 (2017)

23. Eurostat: Internetnutzung durch Privatpersonen - Nahezu acht von zehn Internetnutzern in der EU surften 2016 mit einem Handy oder Smartphone. http://ec.europa.eu/eurostat/documents/29955 21/7771144/9-20122016-BP-DE.pdf/0aba7cec-63d5-411f-ad339dd91aa036e4. Accessed 5 July 2018 (2016)

24. Hill, R., Betts, L.R., Gardner, S.E.: Older adults' experiences and perceptions of digital technology: (dis)empowerment, wellbeing, and inclusion. Comput. Hum. Behav. 48, 415-423 (2015). https ://doi.org/10.1016/j.chb.2015.01.062

25. Tam, M.: A distinctive theory of teaching and learning for older learners: why and why not? Int. J. Lifelong Educ. 33, 811-820 (2014). https://doi.org/10.1080/02601370.2014.972998

26. Venkatesh, V.: Technology acceptance model and the unified theory of acceptance and use of technology. In: Wiley Encyclopedia of Management. American Cancer Society, Atlanta, pp. 1-9 (2015)

27. Davis, F.D.: Perceived usefulness, perceived ease of use, and user acceptance of information technology. MIS Q. 13, 319-340 (1989). https://doi.org/10.2307/249008

28. Venkatesh, V., Morris, M.G., Davis, G.B., Davis, F.D.: User acceptance of information technology: toward a unified view. MIS Q. 27, 425-478 (2003). https://doi.org/10.2307/30036540

29. Brown, S., Venkatesh, V.: (2005) Model of adoption of technology in the households: a baseline model test and extension incorporating household life cycle. MIS Q. 29(3):399-426. https://doi. org $/ 10.2307 / 25148690$

30. Zhou, J., Rau, P.-L.P., Salvendy, G.: Older adults' text entry on smartphones and tablets: investigating effects of display size and input method on acceptance and performance. Int. J. Hum. Comput. Interact. 30, 727-739 (2014). https://doi.org/10.1080/10447 318.2014 .924348 
31. van Biljon, J., Renaud, K.: A qualitative study of the applicability of technology acceptance models to senior mobile phone users. In: Song, IY. et al. (eds.) Advances in Conceptual Modeling-Challenges and Opportunities, pp. 228-237. Springer, Berlin (2008)

32. Mallenius, S., Rossi, M., Tuunainen, V.K.: (2007) Factors affecting the adoption and use of mobile devices and services by elderly people-results from a pilot study. In: 6th Annual Global Mobility Roundtable, vol. 31

33. Kiili, K.: Digital game-based learning: towards an experiential gaming model. Internet High. Educ. 8, 13-24 (2005). https://doi. org/10.1016/j.iheduc.2004.12.001

34. Wang, F., Burton, J.: A solution for older adults' learning of computer skills: the computer game-based learning approach. In: Gibson, D., Dodge, B. (eds.) Proceedings of Society for Information Technology \& Teacher Education International Conference 2010. Association for the Advancement of Computing in Education (AACE), Chesapeake, VA, pp. 2099-2114 (2010)

35. Tews, M.J., Michel, J.W., Noe, R.A.: Does fun promote learning? The relationship between fun in the workplace and informal learning. J. Vocat. Behav. 98, 46-55 (2017). https://doi.org/10.1016/j. jvb.2016.09.006

36. Connolly, T.M., Boyle, E.A., MacArthur, E., Hainey, T., Boyle, J.M.: A systematic literature review of empirical evidence on computer games and serious games. Comput. Educ. 59, 661-686 (2012). https://doi.org/10.1016/j.compedu.2012.03.004

37. Entertainment Software Association: Essential facts about the computer and video game industry. http://www.theesa.com/about -esa/essential-facts-computer-video-game-industry/. Accessed 5 July 2018 (2018)
38. Oh, Y., Yang, S.: Defining exergames \& exergaming. In: Proceedings of Meaningful Play. https://www.researchgate.net/ profile/Stephen_Yang3/publication/230794344_Defining_exerg ames_exergaming/links/0fcfd5047ab31e6cde000000/Definingexergames-exergaming.pdf. Accessed 5 July 2018 (2010)

39. Loos, E.: Exergaming: meaningful play for older adults? In: Zhou, J., Salvendy G. (eds.) Human Aspects of IT for the Aged Population. Applications, Services and Contexts, pp. 254-265. Springer, Cham (2017)

40. Loos, E., Zonneveld, A.: Silver gaming: serious fun for seniors? In: Zhou, J., Salvendy G. (eds.) Human Aspects of IT for the Aged Population. Healthy and Active Aging, pp. 330-341. Springer, Cham (2016)

41. Moreno, R., Mayer, R.E.: Role of guidance, reflection, and interactivity in an agent-based multimedia game. J. Educ. Psychol. 97, 117-128 (2005)

42. Sandford, R., Ulicsak, M., Facer, K., Rudd, T.: Teaching with games. Media Technol. 112, 101-105 (2007)

43. Baur, N., Blasius, J.: Methoden der empirischen Sozialforschung. In: Baur, N., Blasius, J. (eds.) Handbuch Methoden der empirischen Sozialforschung, pp. 41-62. Springer VS, Wiesbaden (2014)

44. Braun, V., Clarke, V.: Using thematic analysis in psychology. Qual. Res. Psychol. 3, 77-101 (2006). https://doi.org/10.1191/14780 88706qp063oa

45. Vinten, G.: Open versus closed questions-an open issue. http://www.ingentaconnect.com/content/mcb/001/1995/00000 033/00000004/art00004. Accessed 19 June 2018 (1995) 